\title{
Educación en Derechos Humanos: Escenarios de Construcción de Paz
}

\author{
Human Rights Education: Peacebuilding Scenarios
}

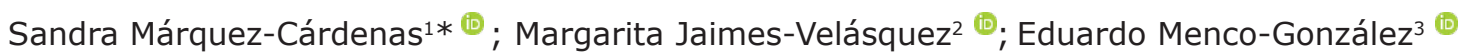

Recibido: 10-10-2020; Aceptado: 15-12-2020; Publicado: 28-12-2020

\section{RESUMEN}

La Educación en Derechos Humanos (EDH) ha sido comprendida y relacionada desde varios factores del desarrollo humano. Además, en Estados en vías de pacificación es clave para la construcción de nuevas ciudadanías. Con el objetivo de realizar un ejercicio investigativo centrado en el análisis de los aportes de la Educación en Derechos Humanos para lograr Escenarios de Paz, presentamos observaciones del último Acuerdo de Paz suscrito por el Gobierno colombiano, atendiendo a sus características de retornar lecciones aprendidas para la construcción de Paz. Se analiza cómo la EDH contribuye a la promoción, garantía y respeto de los Derechos Humanos, declarados universalmente, y cuáles son los contextos del Conflicto armado que se ven trastocados. Por esta razón, el propósito primordial del escrito es la visión desde lo que se denomina Paz Imperfecta frente a la comprensión de consensos que se materializan a través de la EDH, con una mirada especial a la agenda establecida en La Habana (Cuba) y su desarrollo en la mesa de conversación. Las conclusiones encontradas evidencian la relación de los Modelos de Educación en Derechos Humanos, de cara al alcance de lo que se establece en el Acuerdo Final para la terminación del Conflicto y la construcción de una Paz Estable y Duradera. Otra mirada a las conclusiones vislumbra cómo los modelos de EDH son flexibles frente a las exigencias necesarias de los diversos grupos focales, con condiciones y características propias de la sociedad en las que se han desarrollado a consecuencia del Conflicto sociopolítico vivido.

Palabras clave: Educación en Derechos Humanos; Construcción de Paz; Conflicto Armado; Ciudadanía; Escenarios de Paz; Agenda de Paz.

\section{ABSTRACT}

Human Rights Education (HRE) has been understood and related from various factors of human development. In addition, in states in the process of pacification, it is key to the construction of new citizenships. To carry out an investigative exercise focused on the analysis of the contributions of Human Rights Education to achieve Peace Scenarios, we present observations of the last Peace Agreement signed by the Colombian Government, taking into account its characteristics of returning lessons learned for the peace building. It analyzes how HRE contributes to the promotion, guarantee and respect of Human Rights, declared universally, and what are the contexts of the armed conflict that are disrupted. For this reason, the primary purpose of the

${ }^{1}$ Corporación Universitaria del Caribe CECAR, Centro de investigaciones socio jurídicas. Sincelejo, Colombia. sandra.marquez@cecar.edu.co ${ }^{2}$ Corporación Universitaria del Caribe CECAR, Centro de investigaciones socio jurídicas. Sincelejo, Colombia. margarita.jaimes@cecar.edu.co ${ }^{3}$ Corporación Universitaria del Caribe CECAR, Docente catedrático. Sincelejo, Colombia. eduarto.menco@cecar.edu.co 
writing is the vision from what is called Imperfect Peace in the face of the understanding of consensuses that materialize through HRE, with a special look at the agenda established in Havana (Cuba) and its development at the conversation table. The conclusions found show the relationship of the Models of Human Rights Education, in view of the scope of what is established in the Final Agreement for the termination of the Conflict and the construction of a Stable and Lasting Peace. Another look at the conclusions shows how the HRE models are flexible in the face of the necessary demands of the various focus groups, with conditions and characteristics of the society in which they have developed because of the socio-political conflict experienced.

Keywords: Human Rights Education; Peace Building; Armed Conflict; Citizenship; Scenarios of Peace; Peace Agenda

\section{INTRODUCCIÓN}

Los Procesos de Paz son decisiones políticas que asumen los actores armados y el Gobierno para finiquitar una confrontación con daños colaterales (Rios \& Cairos, 2018). En la historia reciente de Colombia, la búsqueda de la Paz ha sido un anhelo que superó varios intentos fallidos (Fisas, 2010); no obstante, el Acuerdo con el Movimiento 19 de Abril (conocido como M-19) provocó una transformación en el modelo del Estado, pasando así de un Estado de Derecho a un Estado Social de Derecho (Congreso de la Republica de Colombia, 1991) fundado en la dignidad humana, la participación real y el bienestar del pueblo ante todo. Este cambio paradigmático acarreó reformas en todos los campos estatales, entre ellas, la Reforma a la Educación (Ley 115 de 1994) que enfatiza su objetivo en el desarrollo de la persona humana como un fin (Art.5).

Igualmente, el Acuerdo de Paz del año 2016, entre el Gobierno Nacional y las Fuerzas Armadas Revolucionarias de Colombia, Ejército del Pueblo - FARC E.P, reafirmó la importancia de la Educación para derribar la exclusión histórica y la pobreza en que ha estado sumergida gran parte de la población (Soler, 2015). También reitera que es necesaria como herramienta para la comprensión, socialización y entendimiento de los tipos de Conflicto que puedan surgir tras la posguerra, la construcción de Escenarios de Paz y la reconstrucción del tejido social, sobre todo en las comunidades donde el acceso a la Educación es escaso.

En este sentido, la Educación en Derechos Humanos es considerada fundamental dentro de los parámetros de la resignificación del pasado y necesaria para afrontar sin violencia los conflictos sociales, económicos y políticos de carácter estructural que han justificado la formación y consolidación de guerrillas desde mitad del siglo XX y lo transcurrido del XXI (Jaimes, 2017). Es decir, que la reconstrucción del tejido social exhorta a la Educación de una ciudadanía activa que convoque a la unidad nacional en torno a la justicia social, a fin de lograr el objetivo principal del Acuerdo de Paz (2016), que ha sido visionado como fundante de una Paz Estable y Duradera (Bolivar, 2016) (Conversaciones, 2016) (Herrera, 2015).

Para ello, según Nikken (1994), es imprescindible reconocer desde la teoría de la EDH que el ejercicio y goce efectivo de estos, como deber del Estado, es un derecho irrenunciable de las personas, que requiere acciones contundentes para su materialización. De acuerdo con Norberto Bobbio (2000), estos derechos requieren ser defendidos, es decir, la mayor preocupación debe centrarse en el cuidado y protección de ellos más que en su justificación, pues "...ahora no se trata tanto de buscar otras razones, sino de poner las condiciones para una más amplia y escrupulosa realización de los derechos proclamados".

\section{LA EDUCACIÓN EN DERECHOS HUMANOS}

Los elementos sustanciales, de la Educación en Derechos Humanos (EDH) han sentado precedentes en formación ciudadana, contribución a la construcción de Paz y cierre de brechas sociales desde su promoción y garantía, y a pesar de los obstáculos para su implementación. Así es como varios investigadores de la materia en América Latina, han coincidido en los derroteros que sustentan la Educación en Derechos Humanos y sus impactos en la formación ciudadana (Magendzo, 2006). 
Márquez-Cárdenas et al - Educación en derechos humanos: Escenarios de construcción de paz

Nancy Flowers (citada por Tibbitts, 2002) la define como: "todo aprendizaje que desarrolle el conocimiento, las habilidades y los valores referentes a los Derechos Humanos" (p. 75). Esta definición está íntimamente arraigada a la promoción y protección de los Derechos Humanos en todos los contextos, siendo la Educación la herramienta idónea para tal fin.

Las Naciones Unidas (1994) exponen que la EDH debe ser un proceso integral de experiencia en todo el desarrollo afectivo, social y político de las personas. Por su parte, lo que estipula el Plan de Acción del Decenio de las Naciones Unidas (1996) para la Educación en Derechos Humanos 1996-2006 es lo siguiente:

El conjunto de actividades de capacitación, difusión e información encaminadas a crear una cultura universal en la esfera de los Derechos Humanos, actividades que se realizan transmitiendo conocimientos y moldeando actitudes, y cuya finalidad es: a) Fortalecer el respeto de los Derechos Humanos y las libertades fundamentales; b) Desarrollar plenamente la personalidad humana y el sentido de la dignidad del ser humano; c) Promover la comprensión, la tolerancia, la igualdad entre los sexos y la amistad entre todas las naciones, las poblaciones indígenas y los grupos raciales, nacionales, étnicos, religiosos y lingüísticos; d) Facilitar la participación eficaz de todas las personas en una sociedad libre; e) Intensificar las actividades de las Naciones Unidas en la esfera del mantenimiento de la Paz. (A/51/506/Add.1, apéndice, Párr. 2).

Como se observa, su intención es que en los Estados se propenda por la construcción de sociedades que reconozcan al otro como un igual dotado de dignidad, libre de toda discriminación y capaz de ser partícipe activo en todos los escenarios de desarrollo de la vida, como la interacción social, los aspectos de la economía y el desarrollo de la democracia participativa. Ahora bien, su desarrollo enfrenta tensiones y problemas no resueltos relacionados a lo polifónico del concepto, al cómo y el dónde, a los discursos ciudadanos de defensa y a los contextos en que se pretende establecer (Magendzo \& Toledo, 2015).

Sin embargo, estas conflictividades surgen de la diversidad de intereses y de los dilemas valorativos que requieren ser derribados en la cotidianidad familiar, comunitaria o social. De ahí que el gran reto de educar desde los Derechos Humanos es contribuir al desarrollo de espacios de convivencia pacífica en el que prime el respeto por los derechos de la otredad (Jaimes, 2015)

\section{LA PAZ COMO DERECHO HUMANO}

En la Constitución Política de Colombia (1991) la Paz es considerada un Derecho Humano con una doble connotación o mirada analítica; es un derecho de ejercicio personal, pero a su vez es un derecho considerado colectivo o de los pueblos (Sentencia C-370, 2006). Esta coexistencia hace que se generen diversos sujetos, además, la persona humana, la sociedad y los estados son titulares de este derecho.

En la Conferencia General de la UNESCO de 1974 (ONU), se concluyó que la Paz no puede entenderse entonces como ausencia de conflictos armados, sino que entraña progreso, respeto mutuo y sobre todo justicia social. Su conceptualización como Derecho Humano es un rechazo a la violencia como forma de relacionamiento. Es un derecho subjetivo exigible que debe ser defendido en todo momento y en todo lugar (Gross, 2005).

La vigencia del Derecho a la Paz impone deberes exigibles a toda persona como el deber de "respecto de la comunidad puesto que sólo en ella puede desarrollar libre y plenamente su personalidad", teniendo en cuenta lo pronunciado en la Declaración Universal de Derechos Humanos en 1948, en el Artículo 29-1 (ONU, 1948), con respecto a la prohibición de toda difusión a favor de la guerra y en el Pacto de Derechos Civiles y políticos, 1969; en el Artículo 20-1 (ONU, 1966).

De lo anterior se concluye que la Paz, además de ser un Derecho Humano, es un principio ético que debe regir las relaciones humanas y de ellos con la naturaleza. Igualmente, es un principio de interpretación de la realidad de una nación y un principio de actuación en el cumplimiento de sus deberes. 
En lo establecido en el Acuerdo Final para la terminación del Conflicto y la construcción de una Paz Estable y Duradera, se tiene presente lo que estipula el Artículo 22 constitucional (Congreso de la Republica de Colombia, 1991), el cual argumenta que la Paz es un derecho y deber de obligatorio cumplimiento, anudando esta declaración con lo que se afirma en el Artículo 95 de la Constitución Política de la República de Colombia frente al ejercicio y desarrollo de los derechos y las libertades reconocidas en la Constitución, lo que finalmente sería la base para el reconocimiento de responsabilidades y el logro de Escenarios de Paz.

Los acuerdos que conforman el nuevo Acuerdo Final contribuyen a la satisfacción y desarrollo progresivo de derechos fundamentales (Conversaciones, 2016); en este sentido, se atribuye la garantía y promoción de los Derechos Humanos desde el ejercicio de la Paz, y los consensos a partir de los disensos, como bien se ha decantado en el desarrollo del ciclo de conversaciones surtido en La Habana (Cuba).

\section{APORTES DE LA EDUCACIÓN EN DERECHOS HUMANOS A LA CONSTRUCCIÓN DE PAZ}

Lo que se ha estipulado en Educación con enfoque de Derechos Humanos, encuentra su genealogía en las guerras civiles, guerras intestinas, periodos de dictadura, violencia estructural e institucionalizada, en voz de Abraham Magendzo (2006. p.11) los inicios del trasegar de la Educación en clave de Derechos Humanos se estarían pensado como respuesta para recobrar la democracia, terminar las guerras, estabilizar el Estado golpeado por esta clase conflictos, cuya característica es ser armado y violar sistemáticamente los Derechos Humanos. Sosteniendo así que sus planteamientos vendrían desde el fundamento ético de un nuevo paradigma, cuyo eje seria la Educación para la ciudadanía.

Los espacios de formación eran escasos por no decir que nulos, se tornaba peligroso hablar de Derechos Humanos y mucho más relacionarlo con la Educación.

Es necesario reafirmar que la Educación popular representó un fuerte cimiento para lo que después conoceríamos como Educación en Derechos Humanos; esos pequeños espacios que se gestaban, liderados por activistas de Derechos Humanos representaron para varios países, sobre todo de América Latina, el logro de incluir en los currículos el marco legal, ético y filosófico de los Derechos Humanos.

En su evolución, se plantea entonces el enfoque desde la mirada transversal de los Derechos Humanos en los niveles de aprendizaje, representando también la inserción de temas fundantes, alternos y conectados con los Derechos Humanos, en este sentido se plantea la inserción de temas como el derecho al medio ambiente, el respeto a la multiculturalidad, el reconocimiento de la sexualidad y los derechos sexuales y reproductivos, la progresividad del derecho a la salud, la formación ciudadana, la convivencia pacífica y la democracia. Estos ejes representarían en la solución a las nuevas conflictividades la oportunidad de transformación del Conflicto hacia Escenarios de Paz.

La Educación para la Paz, por su parte, fue considerada una corriente pedagógica dentro del referente de la Escuela Nueva (González, Hernández, \& Valle, 2009); sin embargo, así como sucedió con la Educación en Derechos Humanos, su génesis tendría lugar en el análisis de las consecuencias de lo sucedido en la primera y segunda guerra mundial, si bien se abanderaba la Educación para la democracia, no es menos cierto que este sería posteriormente uno de los ejes de trabajo de lo planteado desde la Educación en Derechos Humanos y su articulación con la Educación para la Paz.

Sin embargo, la propuesta de Escuela Nueva, encontraba limitaciones teóricas, lo que representó la reducción de su permanencia en el escenario pedagógico en apenas tres décadas; causas vistas desde el entorno político de los países que ya se reconocían como potencias, para Estados Unidos de América, por ejemplo, representaba cambios y tensiones entre lo político y el costo de la enseñanza en Educación para la Paz, sin negar que frente a estas resistencias encontrábamos la imposición de ideologías desde los espacios de enseñanzas. 
Ante este panorama, visto en otra esfera mundial, como Europa, encontramos nuevas ideas educativas, por ejemplo, la italiana María Montessori (Ospina, 2010) o la de Adolphe Ferrière, quienes plantearon la transformación de las instituciones educativas de la posguerra, a partir de la aplicación de métodos de enseñanza que más tarde sentarían las bases del desarrollo conceptual y práctico de la Educación para la Paz, se pretendía brindar al ser humano en la niñez la posibilidad de alcanzar el activismo principal en el proceso de aprendizaje, a través de la expresión de sus emociones, el desarrollo autónomo de capacidades, las cuales entrarían a permitir su relación y crecimiento desde el entorno, asimismo, estaría en la capacidad de replicar lo aprendido en diferentes etapas de su crecimiento.

Con estas referencias históricas nace la clasificación de la Educación para la Paz, dentro de las propuestas integradoras que permiten la aprehensión de la Educación para la Paz frente a la transformación social de la Educación en Derechos Humanos, encontramos la reseña de Sophia Herrero Rico, sobre Xesus R. Jares que en su producción "Educación para la Paz su teoría y práctica" (Rico, 2003) propone las etapas correspondientes a la Educación para la Paz, como garante del ejercicio de los Derechos Humanos y su concordancia con la Educación en Derechos Humanos. En síntesis, presenta cuatro etapas para la comprensión de su desarrollo histórico:

La Escuela Nueva, la cual recoge lo propuesto en el periodo de la primera posguerra mundial, dónde el hábito sería el reconocimiento de esos derechos trastocados por los hechos acontecidos.

Educación para la Paz propuesta desde la UNESCO, como organismo especializado de las Naciones Unidas, cuyo nacimiento es después de la Segunda Guerra Mundial (1945). Aquí se estipula, como objetivo principal de mandato, la promoción de una cultura de Paz mediada de manera imperativa desde la Educación, apoyando a los Estados Miembros para que impartan y promuevan desde sus funciones de cuidado al sujeto como receptor de normas de Derecho Internacional Público, una Educación de Calidad, la cual debe plantearse desde la inclusión, lo que permitirá el cumplimiento a los postulados respectivos de Paz y Derechos Humanos. Entre otros ejes se establece la tolerancia, el entendimiento intercultural, la democracia, la no violencia y el respeto. (ONU, 2011). Se hace importante mencionar que aquí es determinante la relación que se devela, partiendo de la propuesta en la genealogía de la Educación en Derechos Humanos, es decir, las sinergias de ambos enfoques pedagógicos que comparten las bases teóricas de democracia, multiculturalidad y respeto.

La No-violencia, como imperativo de la Educación para la Paz, representado por los postulados de Gandhi en su legado de pedagogía de la esperanza en una sociedad sin violencia (Rico, 2003).

Otra fase de la Educación para la Paz sería denominada investigación para la Paz, de donde emerge Paz negativa, Paz positiva, el empoderamiento pacifista y la cultura de Paz, se destacan las aportaciones de Johan Galtung, para darle el toque innovador al enfoque de Educación para la Paz (Jarés, 1999), partiendo de la importancia del empoderamiento desde las bases sociales y culturales.

Así, la nueva propuesta frente a los aportes de la EDH y a la construcción de Paz vislumbraría la innovación social con razones de peso de diversa índole, incluyendo la imposición legal a los Estados para la garantía de educar y de generar desde la escolaridad espacios de Paz, donde los ejes comunes seguirían siendo: el medio ambiente, la democracia, la participación política y la justicia social.

Los marcos evolutivos de las estrategias de la Educación para la Paz y los ejes de los Derechos Humanos se han representado a través de la construcción de la ciudadanía como base de convivencia, lo que ha permitido observar desde la conflictividad espacios para solucionar las controversias sin actos violentos.

Jean Paul Lederach (1984) propone que la Educación para la Paz debe contribuir a la transformación pacifica de los conflictos, proyectar herramientas a las nuevas generaciones para que aprendan que la violencia no es la única salida para solucionar conflictos de cualquier índole. 
La EDH en contextos de violencia socio política, como el caso de Colombia, representa uno de los desafíos para romper con la exclusión de grupos poblacionales históricamente discriminados, los cuales se han empoderado pacíficamente desde los planteamientos de la Educación para la Paz. Cerrar la brecha de violencia contra las mujeres, reducir la discriminación por sexo, por identidad u orientación sexual, representa para los desafíos de la cultura de Paz, la garantía del desarrollo humano y el impacto rápido en el cambio social (Márquez, 2018).

El carácter transversal de la EDH ha permitido avanzar en el reconocimiento de grupos minoritarios, entre estos los étnicos, generando un currículo flexible que permite a partir de la Educación en Derechos Humanos analizar los derroteros que han demarcado la Etnoeducación, teniendo en cuenta, entre otras, las luchas de los grupos étnicos por el cuidado, mantenimiento y la protección del medio ambiente (Rojas, 2011). De esta manera, se plantea la interculturalidad basada en lo que propone la Educación en Derechos Humanos, garantizando desde este paradigma la declaración reconocida a grupos minoritarios.

Desde la esfera de la participación democrática, la EDH aporta a espacios de Paz y a la garantía del goce efectivo de los derechos civiles y políticos de todas las personas sin distinción; el punto dos del Acuerdo Final, suscrito por el Estado Colombiano con la guerrilla de las FARC EP, es muestra de la apertura democrática para construir la Paz. (Conversaciones, 2016, pág. 35). Se propone dentro de estas correlaciones distribuir de manera equitativa los recursos públicos, que en términos de Paz es la importancia a la construcción de lo público como garante del desarrollo del Estado Social de Derecho, la modernización del régimen electoral, el ejercicio de los derechos democráticos y la participación ciudadana en todas las etapas de la planeación territorial, permitiendo así lograr en los territorios, históricamente afectados por el Conflicto, la representación en corporaciones públicas de elección popular.

Reafirmando la correspondencia entre EDH y sus aportes a la construcción de Paz, se infiere como la justicia social se materializa desde las garantías y ejercicio de estos, libre de cualquier tipo de violencia, sin desconocer estadios de Conflicto que pueden ser transformados pacífica y creativamente (Fisas, 2011).

Dentro de los antecedentes de lo que se ha planteado como Educación en Derechos Humanos a nivel del Estado Colombiano y en etapas previas a la llegada de los equipos negociadores a La Habana, cabe mencionar el Plan Decenal de Educación 2006-2016, el cual sirve de observancia dinámica para el desarrollo educativo del país, permitiendo a partir de sus postulados el reconocimiento de la función social de la Educación, la cual explora en los y las aprendices como seres humanos la participación activa frente a la promoción de derechos, se atiende de manera especial el enfoque territorial y las particularidades de los contextos, desde lo local, pasando lo regional hasta lo nacional y el reconocimiento internacional. Al observar de manera integral estos constructos, se infiere la importancia de la transformación de la realidad social, política y económica del país, para logro de la Paz, la superación de índices de pobreza y de exclusión, la reconstrucción del tejido social, el fomento de los valores democráticos, y la formación de ciudadanos libres con valores de solidaridad y autónomos. Lo anterior da lugar al respeto y promoción de los Derechos Humanos cuyos ejes giran en el aprendizaje de temas como la diversidad étnica, las libertades religiosas, políticas y de opción de género, entre otros (Ministerio de Educación Nacional, 2006).

Los Derechos Humanos, desde la Educación involucran una práctica pedagógica considerada innovadora y a la vez cuestionadora de parte de todas las personas que convergen en este punto, entre estos se han analizado la tendencia desde: maestros, alumnos, cuerpo directivo, padres/ madres de familia y la comunidad en general (Marín, 2012), de allí la importancia de introducir nuevos conceptos y metodologías activas, que permitan al maestro desarrollar sus conocimientos y al estudiantes aprehender los conocimientos, a partir de lo planteado en el marco ético de los Derechos Humanos como garante en la construcción de Paz. Cabe recordar que el Acuerdo de Paz suscrito por el Gobierno de Colombia en 2016 representa la comprensión de estos postulados de la Educación en Derechos Humanos desde escenarios de desacuerdos, convertido en acuerdos suscritos de Paz con múltiples observaciones que permiten el desarrollo del ser humano a partir del reconocimiento de sus derechos. 
El Plan Nacional de Educación en Derechos Humanos, como hoja de navegación formulada por el Ministerio de Educación de la República de Colombia, contempla las siguientes recomendaciones en relación con la EDH y su afinidad con los espacios de Paz propicios para la articulación de los ejes de acción:

Desligar, a la escuela del Conflicto, concretarla y protegerla como "espacio de Paz". La escuela es por esencia un espacio para la cimentación permanente de sujetos de derechos, siendo así el escenario idóneo para educar para y en la Paz a los discentes. En ella, el aprendiz debe ser dotado de las habilidades para resolver los conflictos propios de la académica desde mecanismos alternativos de conflictos. Es ella el espacio favorable para la aprehensión de los ejes nucleares con enfoque de Derechos Humanos, sumando a esto que se propicia su análisis crítico. Igualmente, es importante destacar el rol del educador o educadora que debe estar convencido del papel transformador de los la EDH. Por otro lado, implica que el Estado debe garantizar el respeto en las aulas y el cumplimiento del DIH. Asimismo, sobre todo en los entornos rurales, garantizar la continuidad del maestro y evitar los riesgos y amenazas contra la vida del docente.

Es necesario legitimar, desde la Educación los Derechos Humanos el desarrollo de estrategias de enseñanza-aprendizaje, desde las voces de los defensores de Derechos Humanos, del personal docente y de las vivencias propias de alumnos para adaptar, la flexibilidad del proceso educativo al contexto colombiano. El cumplimiento por parte del Estado de los instrumentos internacionales se da en varias vías, el cual tiene en cuenta las múltiples personalidades que participan en el proceso educativo. Esto permite que la Educación en Derechos Humanos, cumpla con objetivos integrales para pasar de la teoría del aprendizaje a la práctica cotidiana, lo cual se ha conocido como aprendizaje basado en problemas.

Establecer mecanismos y herramientas que hagan efectiva la eliminación de toda discriminación de las niñas embarazadas y niñas-madres al interior del ambiente de aprendizaje, como lo postuló la Corte Constitucional. Si bien el tema de embarazaos a temprana edad es una arista alterna a la Educación en Derechos Humanos, no es menos cierto que eliminar, a través de acciones afirmativas toda discriminación de las niñas embarazadas y niñas - madres, como objetivos misionales de educar en Derechos Humanos.

Múltiples recomendaciones han surgido en materia de Educación de los Derechos Humanos, es así como en 1995, se recomendó desde el Comité de Derechos Económicos, Sociales y Culturales al Estado impartir Educación sobre este tema en todos los niveles de enseñanza - aprendizaje, haciendo un especial énfasis en los contenidos nucleares que se imparten en la Educación primaria o elemental. Esta recomendación, solicita de manera especial al Gobierno otorgar prioridad a la integración de la Educación en Derechos Humanos a los programas y planes de estudio de los colegios y universidades del país; sin embargo, a pesar de la existencia de estos múltiples instrumentos y la infinidad de recomendaciones, se sigue debatiendo y luchando para que todo lo dicho y escrito sea una realidad en el progreso humano y en la formación de la ciudadanía.

Ahora bien, al realizar el análisis documental del alcance del Acuerdo Final gobierno de Colombia - FARC-EP de cara a lo que hemos desarrollado sobre EDH y su aporte a la construcción de $\mathrm{Paz}$, tenemos desde los puntos concertados que: vislumbrar la reforma rural integral (punto 1 de la agenda) como solución a un problema histórico en Colombia y devolverle al campo su valor, representara la aplicación de estrategias educativas enfocadas a colectivos campesinos que a la vez pueden ser víctimas del Conflicto o también estar en procesos de reintegración y/o reincorporación a la vida civil, con enfoque étnico, mujeres ruarles entre otras condiciones propias de la negoción en medio del Conflicto.

Aceptar la participación política como un Derecho Humano de esas personas que pertenecieron a la guerrilla de las FARC y que ahora afrontan la reincorporación a la vida civil, representaría para la Educación en Derechos Humanos uno de los desafíos latentes propios del todo acordado, la relación aquí entre la Educación y la Paz se ve concreta en lo que planteábamos en renglones anteriores sobre la evolución del concepto de Paz. 
El fin del Conflicto, quizá el punto de la agenda más difícil de negociar, encarno la necesidad de tomar las bases de la EDH propuestas a través de la historia y materializadas en Colombia por múltiples leyes que permiten la comprensión de los alcances del mismo, es así como al entrar en vigencia la ley 1620 de 2013, a través de la cual se crea el "Sistema Nacional de Convivencia Escolar y Formación para el ejercicio de los Derechos Humanos, Sexuales y Reproductivos y la prevención y mitigación de la Violencia Escolar" (2013). En este orden igualmente con la Ley número 1732 de 2014, por la cual se establece la "Catedra de la Paz" (2014) en todas las Instituciones Educativas del país, podemos realizar un análisis desde los hechos que habían acontecido y de la realidad que ahora se vivía, como retos desde la EDH, para la construcción de Paz; de estas múltiples observancias, la Escuela es el escenario idóneo para comprender los alcances de lo que la agenda de Paz desarrollaba, indistintamente de la postura política, social, económica y/o cultural, estábamos entonces frente a anhelos propios construidos desde la Paz imperfecta.

La solución al problema de las drogas ilícitas, tema propuesto en la agenda de negociación, suscitada en La Habana (Cuba) por primera vez se trataba, para dar respuesta al fin del Conflicto, represento esfuerzos invaluables desde la Educación para comprender temas en clave de Derechos Humanos como la vocación de la tierra, el derecho al trabajo, el enfoque diferenciador y territorial, la garantía de acceso a derechos económicos, sociales y culturales por parte del Estado en territorios donde la ausencia era notoria. Con la enseñanza del Acuerdo de Paz en Colombia, a medida que se concretaba en La Habana, se fue realizando desde la pedagogía, la comprensión del enfoque de los Derechos Humanos intrínsecamente implícitos, que develan sobre todo su respeto y promoción.

Las víctimas en el proceso de socialización de los alcances del Acuerdo de Paz, es el grupo focal que representa el eje o la columna vertebral de lo pactado y representa el escenario de construcción de Paz permanente tanto en la órbita social como política y territorial. El acuerdo estable el sistema de justicia, verdad, reparación y garantías de no repetición como pilares propios de justicia transicional cuya relación es inherente a la EDH.

Por último, el sistema refrendación acordado por el equipo negociador en La Habana, nos referimos al plebiscito del 02 de octubre o también conocido como "plebiscito por la Paz" en 2016, requirió de manera urgente y tajante la articulación de los postulados de Educación en Derechos Humanos sobre todo en todo en los grupos poblacionales, entre estos los y las jóvenes mayores de 18 años habilitados para ejercer su Derecho Humano a elegir; por la naturaleza del mecanismo de participación ciudadana utilizado fue necesario que las estrategias de enseñanzaaprendizaje de Educación en Derechos Humanos se representaran desde los distintos niveles de espacios educativos y desplegarlas hacia las zonas rurales en donde están asentadas las comunidades más afectadas por el Conflicto armado. Su desenlace no será objeto en este escrito, sin embargo, podemos rescatar que fue la EDH en la que nos permitió comprender el alcance de la construcción de ciudanía como garante de Paz.

La revisión de lo pactado se hace desde la misión de verificación al Acuerdo de Paz, así lo establece el punto 6 del Acuerdo de Paz y está a cargo de las Organización de las Naciones Unidas, del Gobierno de Colombia y de las FARC en proceso de reincorporación a la vida civil. Se crea un sistema integrado que permite la verificación de información el cual requiere en la actualidad de las plataformas de Educación en Derechos Humanos para darle objetividad y calidad al avance de lo pactado y la observancia de desafíos que se presentan, sin distinción de fijación social, económica o política. Aquí el objeto al que Morin hace alusión hace parte de ese todo para Escenarios de Paz y Reconciliación.

\section{CONCLUSIÓN}

Al inferir que el Conflicto armado en Colombia ha dejado secuelas negativas sobre la comunidad que lo sufre y en los territorios donde suceden los hechos, los Procesos de Paz han sido escenarios propicios para empezar a generar espacios reflexivos de reconstrucción del tejido social donde la EDH ha logrado sentar bases necesarias para su comprensión y sobre todo su utilidad. 
Se enfatiza que el Mandato Constitucional Colombiano es claro en su Artículo 67, al declarar la Educación como un servicio público, que tiene una función social definida, para el cumplimiento de este precepto, se establecen las líneas estratégicas desde lo propuesto en la Política de Educación en Derechos Humanos, a través del Plan Nacional de Educación en Derechos Humanos (PLANEDH) a partir de este documento y en concordancia con los instrumentos internacionales, en el año 2013, con la Ley 1620, se crea el Sistema Nacional de Convivencia Escolar y Formación para el ejercicio de los Derechos Humanos, la Educación para la sexualidad y la prevención y mitigación de la violencia escolar. Es necesario tener la claridad de este precedente sobre todo en el contexto en el que traemos los ejemplos de construcción de Paz con miras a la reparación integral de todos los actores del Conflicto.

La contribución de la EDH a la construcción de Paz ha establecido criterios validos como: la necesidad de contacto entre grupos focales con la finalidad de restablecer relaciones destruidas, especialmente después de décadas de Conflicto Armado, reconociendo que, en el territorio, Escuela, espacio de formación, o espacio no formal de dialogo pueden converger víctimas del Conflicto, personas reintegradas o en proceso de reintegración y/o miembros de la fuerza pública y la sociedad civil. Un ejemplo es lo analizado en la Política Integral de Derechos Humanos y Derecho Internacional Humanitario, la cual establece en los soportes teóricos los ejes transversales, objetivos definidos e inclusivos y los fundamentos para la capacitación en Derechos Humanos y derecho internacional humanitario, así mismo presenta los programas que en estos núcleos temáticos deben conocer y desarrollar al interior de las fuerzas armadas y la fuerza pública, para su puesta en práctica en el desarrollo de sus operaciones; esta estrategia es fundamental dentro de Escenarios de Paz precisamente por los actores (participantes) que confluyen y su observancia como agentes del estado.

Si bien el Estado Colombiano adopta modelos de EDH según estándares propuestos desde la ONU y en relación a su responsabilidad frente a la protección y garantías de los mismos no es menos cierto que la efectividad de la Educación en Derechos Humanos va a depender de factores alternos como las consecuencias del prolongado Conflicto Armado interno, lo cual conlleva a la adaptación del modelo de Educación en Derechos Humanos a enfoques diferenciales, territoriales y a combinaciones internas y externas que permitan el desarrollo humano en Escenarios de Paz.

Los aportes de la EDH han permitido desde varios ejes la construcción de ciudadanía participativa, más comprometida en el respeto de las diferencias y entendiendo la Paz más allá de un proceso como aquel conceso de vivir en armonía aun con conflictos alternos.

El enfoque territorial demuestra que la construcción de Paz es diversa, la cual vincula la promoción y protección de los Derechos Humanos en cualquier ciclo vital y que no es solo la Escuela es el campo de apropiación del conocimiento, sino que, por el contrario, de la cotidianidad nace las expresiones de construcción de Paz imperfecta.

La socialización de la agenda propuesta en La Habana, la cual recoge experiencias de otros procesos Paz no hubiese llegado al colectivo nacional sino utilizamos modelos de Educación en Derechos Humanos que convergen con la realidad que vivimos. Estos modelos de Educación al ser flexibles permiten su adaptación al grupo focal que por derecho debe conocer los alcances de lo que pacto en La Habana Cuba en el año 2016.

La necesidad de educar en Derechos Humanos, desde el ciclo vital representará un trabajo continuo en la comprensión de lo sucedido a razón del Conflicto armado, el anhelo de Paz desde la individualidad, hasta la colectividad y la constante transformación pacífica de las nuevas conflictividades que se puedan presentar.

La Educación en Derechos Humanos tiene la posibilidad de evolucionar y adaptarse al grupo focal de aprendizaje, así como a las estrategias que proponga el orientador no obstante su contribución a escenarios de construcción de Paz va a tener disensos y consensos que permitirán ir materializando el querer de los Derechos Humanos en su respeto y promoción. 
Nos encontramos en momentos inquietantes donde la construcción de Paz, aun con otros actores y factores del Conflicto otorgan el valor nominal a la EDH, como aporte válido y permanente en la comprensión de los derechos de la otredad, los cuales fortalecen la práctica ciudadana desde el ejercicio y goce de los Derechos Humanos.

\section{REFERENCIAS}

Bobbio, N. (2000). El problema de la guerra y las vías de la Paz. Barcelona: GEDISA.

Bolivar, A. (2016). Educar democráticamente para una ciudadanía activa. Revista Internacional de Educacion para la justicia social 5.1, 69-87. Obtenido de https://repositorio.uam.es/ bitstream/handle/10486/671304/RIEJS_5_4.pdf?sequence=1

Congreso de la Republica de Colombia. (1991). Constitución Política de Colombia. Obtenido de https://bibliotecadigital.ccb.org.co/bitstream/handle/11520/24627/CONSTITUCION\%20 POLITICA\%201991.pdf?seq

Constitucional, C. C. (2006). Sentencia C-370. Obtenido de https://www.corteconstitucional.gov. co/relatoria/2006/C-370-06.htm

Constitucional, C. C. (2013). Ley No 1620. Por la cual se crea el Sistema Nacional de Convivencia Escolar y Formación para el Ejercicio de los Derechos Humanos, la Educación para la Sexualidad y la Prevención y Mitigación de la Violencia Escolar. Obtenido de https://www. mineducacion.gov.co/1759/articles-327397_archivo_pdf_proyecto_decreto.pdf

Constitucional, C. C. (2014). Ley 1732. Por la cual se establece la Cátedra de la Paz en todas las instituciones educativas del país. Obtenido de https://www.funcionpublica.gov.co/eva/ gestornormativo/norma.php?i=59313

Conversaciones, M. d. (2016). ACUERDO FINAL para la TERMINACIÓN DEL CONFLICTO \& LA CONSTRUCCIÓN DE UNA PAZ ESTABLE Y DURADERA. BOGOTA: OFICINA DEL ALTO COMISIONADO PARA LA PAZ. Obtenido de https://www.jep.gov.co/Marco\%20Normativo/ Normativa_v2/01\%20ACUERDOS/Texto-Nuevo-Acuerdo-Final. pdf?csf =1\&e=0fpYA0

Fisas, V. (Noviembre de 2010). El proceso de Paz en Colombia. El proceso de Paz en Colombia(17), QUADERNS DE CONSTRCCIO DE PAU . Barcelona, España: Escola de Cultura de Pau. Recuperado el 15 de julio de 2020, de http://ibdigital.uib.es/greenstone/collect/cd2/import/ escolaculturapau/ecp0022.pdf

Fisas, V. (2011). Educar para una cultura de Paz. Quaderns De Construcció de Pau, 2-8. Obtenido de https://www.asproul.org/wp-content/uploads/2016/07/educar_cultura_Paz.pdf

González, P. L., Hernández, D., \& Valle, E. (2009). Acerca de los antecedentes de la Educación para la Paz. MENDIVE Revista Científico pedagógica, 108-111. Obtenido de https://mendive. upr.edu.cu/index.php/MendiveUPR/article/view/343/341

Gross, H. (2005). El Derecho Humano a la Paz. Anuario de Derecho Constitucional Latinoamericano. Recuperado el 15 de julio de 2020, de http://corteidh.or.cr/tablas/R21744.pdf

Herrera, R. (28 de abril de 2015). Entrevista a Ricardo Herrera, vocero del Congreso de los Pueblos: "La Paz es justicia social, la Paz son cambios estructurales. No es únicamente el silenciamiento de las armas.". (L. A. perez, Entrevistador) Obtenido de http://www.memoria. fahce.unlp.edu.ar/art_revistas/pr.6688/pr.6688.pdf 
Márquez-Cárdenas et al - Educación en derechos humanos: Escenarios de construcción de paz

Jaimes, M. (2015). El teatro y la Educación en Derechos Humanos. Una estrategía para analizar la Educación. Reencuentro, 148-160. Obtenido de https://reencuentro.xoc.uam.mx/index. $\mathrm{php} /$ reencuentro/article/view/887

Jaimes, M. (2017). La educacion no formal en Derechos Humanos. En P. Guzman, Tendencias Nacionales e Internacionales en la formación de abogados (págs. 203-219). Barranquilla: Universidad Simon Bolivar. doi: https://doi.org/10.21892/9789585430273

Jarés, X. (1999). Educación para la Paz. Su teoría y su práctica. Madrid: Popular.

Lederach, J. P. (1984). Educar para la Paz. En J. P. Lederach. Fontamara. Obtenido de https:// www.asproul.org/wp-content/uploads/2016/07/educar_cultura_Paz.pdf

Magendzo, A. (2006). Educación en Derechos Humanos: Un desafío para los docentes de hoy. Santiago de Chile: LOM.Concha y Toro 23. Santiago.

Magendzo, A., \& Toledo, M. I. (3 de septiembre de 2015). Educación en Derechos Humanos: Estrategia Pedagógica-Didáctica centrada en la controversia. Revista electronica EDUCARE, 1-16. doi: http://dx.doi.org/10.15359/ree.19-3.2

Marín, A. H. (2012). Educar en Derechos Humanos: una tarea urgente Human Rights Education: and urgent task. doi:https://doi.org/10.21500/01235826.1784

Márquez, S. M. (2018). La pedagogía para la Paz en la formación de personas con orientaciones sexuales e identidades de género no hegemónicas victimas del Conflicto armado.Una mirada desde las narrativas de la memoria. En P. Gentili, Cuadernos de Investigación en Educación (págs. 93-107). Bogotá: CEDALC. Obtenido de http://biblioteca.clacso.edu.ar/clacso/ otros/20200218024737/Cuadernos-de-Educacion-Vol-2.pdf\#page=53

Ministerio de Educación Nacional. (20 de septiembre de 2006). Plan Nacional Decenal de Educación 2006-2016. Plan Nacional Decenal de Educación. Bogotá, Colombia: USAID-OIM. Obtenido de https://www.minsalud.gov.co/sites/rid/Lists/BibliotecaDigital/RIDE/INEC/IGUB/Plan\%20 decenal\%20de\%20educacion\%202006\%20-\%202016.pdf

Muñoz, F. A., \& Arenas, J. M. (2018). El Giro Epistemológico y Ontológico en la Investigación para la Paz. En L. D. Escobar, Empoderamiento Pacifista (págs. 19-57). Medellin - Colombia: Universidad de Medellin.

Nikken, P. (1994). El concepto de Derechos Humanos. En P. Nikken, Estudios basicos de Derechos Humanos (págs. 15-37). San Jose: IIDH. Obtenido de http://datateca.unad. edu.co/contenidos/90150/Curso_AVA/Curso_AVA_8-02/Entorno_de_Conocimiento_8-02/ Bibliografia_Unidad_2/Concepto_de_Derechos_Humanos.pdf

Nin, M. C., \& Lorda, M. A. (2019). Políticas Educativas que propician la educacion en memoria y Derechos Humanos. Revista Universitaria de Geografía, 28. Obtenido de https://www. redalyc.org/jatsRepo/3832/383260260006/383260260006.pdf

ONU. (1948). Declaración Universal de Derechos Humanos. Paris. Obtenido de https://www. ohchr.org/EN/UDHR/Documents/UDHR_Translations/spn.pdf

ONU. (1966). Pacto de Derechos Civiles y políticos. En S. Tratatos (Ed.)., (pág. 171). Recuperado el 28 de Julio de 2020, de //www.refworld.org.es/docid/5c92b8584.html

ONU. (1996). ASAMBLEA GENERAL DE LAS NACIONES UNIDAS; INFORME DEL ALTO COMISIONADO DE LAS NACIONES UNIDAS PARA LOS DERECHOS HUMANOS. NEW YORK: NACIONES UNIDAS. 
ONU. (11 de Marzo de 2011). Organización de las Naciones Unidas para la Educación, la Ciencia y la Cultura; Servicio de prensa UNESCO. Obtenido de http://www.unesco.org/new/es/mediaservices/single-view/news/unesco_promotes_a_culture_of_peace_through_education/

ONU. (s.f.). Oganización de las Naciones Unidas para la Educación, la Ciencia y la Cultura: Servicio de Pensa- UNESCO. Recuperado el 11 de Marzo de 2011, de http://www.unesco.org/new/ media-service/single-view/unesco_promontes_a_culture_of_peace:through_education/

Ospina, J. (11 de Enero de 2010). La Educación para la Paz como respuesta ético-pollítica de emancipación democrática. origen, fundamentos y contenidos. Universitas: Revista de filosofía, Derecho y Política., 93-125. Obtenido de http://hdl.handle.net/10016/8897

Rico, S. H. (2003). Reseña de "Educación para la Paz. Su teoría y su práctica" de Xesús R. Jares. Convergencia. Revista de Ciencias Sociales,, 285-298. Obtenido de https://www.redalyc. org/pdf/105/10503313.pdf

Rios, J., \& Cairos, H. (2018). Los Discursos sobre la participación política en el proceso de Paz de Colombia. Araucaria. Revista Iberoamericana de Filosofia, Política y Humanidades, 317-339. Obtenido de https://dialnet.unirioja.es/servlet/articulo?codigo $=6389576$

Rojas, A. (2011). Gobernar (se) en nombre de la cultura. interculturalidad y Educación para grupos étnicos en colombia. Revista Colombiana de antropologia, 173-198. Obtenido de https://dialnet.unirioja.es/servlet/articulo?codigo $=6354117$

Soler, D. G. (2015). "La Educación como una garantía de no repetición en tiempos de negociación de la Paz". Trabajo Social:18, 57-54. Obtenido de https://revistas.unal.edu.co/index.php/ tsocial/article/view/58571/56281

Tibbitts, F. (2002). Nuevos modelos de educación en derechos humanos. DEHUIDELA, 75-80. Obtenido de https://www.corteidh.or.cr/tablas/r24461.pdf 